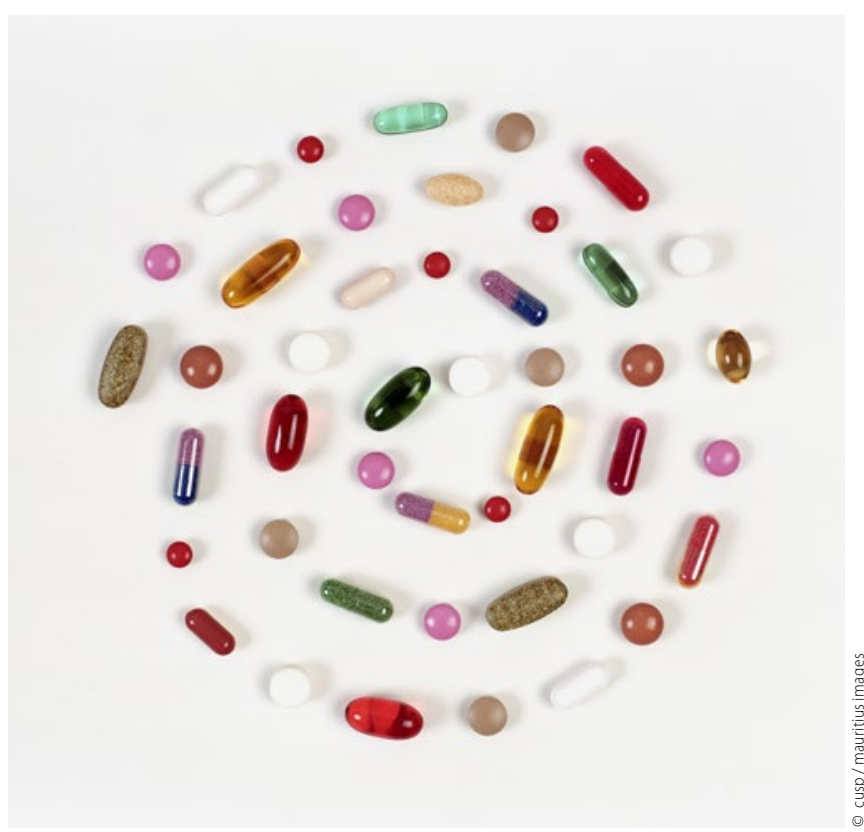

Forscher entwickeln neuen Wirkstoff

\section{Schmerzmittel ohne uner- wünschte Nebenwirkungen}

Opioide werden insbesondere bei Schmerzen durch Gewebeverletzungen und Entzündungen eingesetzt, beispielsweise nach Operationen, bei Arthritis oder bei Tumorerkrankungen. Häufige, zum Teil gefährliche Nebenwirkungen sind Benommenheit, Übelkeit, Verstopfung oder sogar Atemstillstand. Wissenschaftlerinnen und Wissenschaftler der Freien Universität Berlin und der Charité haben eine neue Klasse von Schmerzmedikamenten entwickelt. Mittels neuartiger chemischer Synthesemethoden koppelten Berliner Forscher das konventionelle Opioid Morphin an Trägermoleküle, sogenannte Nanocarrier. Für das auf diese Weise neuentwickelte Polymerkonjugat „Polyglycerol-Morphin“ konnten die Wissenschaftler eine Schmerzstillung ausschließlich im entzündeten Gewebe nachweisen und damit das Risiko bedrohlicher Nebenwirkungen in gesunden Geweben wie im Gehirn oder in der Darmwand ausschalten.

Die Forschungsergebnisse wurden in der jüngsten Ausgabe des Fachmagazins eLife veröffentlicht.

mar

\section{BZÄK-Video}

\section{Keine Angst vor HIV, HCV und HBV}

In einem Video, das sich an Zahnmedizinische Fachangestellte richtet, klärt die Bundeszahnärztekammer auf, dass viele Sorgen vor einer Infektion mit HIV oder Hepatitis B oder C bei der Berücksichtigung der geltenden Hygienestandards im Behandlungsalltag unbegründet sind.

Es sei normal, wenn man bei der ersten Behandlung eines infizierten Patienten am Anfang etwas unsicher ist, heißt es in dem Video. „Grundsätzlich gilt: Die einzige Flüssigkeit, die HIV übertragen könnte, ist Blut", erklärt das Video, und auch dafür sei eine ausreichende Menge Viren, die über Blut-zu-Blut-Kontakt in den Körper gelangen, nötig. Heutzutage würden Medikamente die Virenanzahl aber so weit senken, dass es nicht mehr zum Ausbruch von Aids kommen könne. Die Wahrscheinlichkeit einer Übertragung sei daher sehr gering. Gegen Hepatitis B schütze eine Impfung.

Gegen Hepatitis $C$ gebe es keine Impfung, eine Übertragung sei wie bei HIV möglich, aber unwahrscheinlich. Sollte es doch zu einer Kontamination kommen, beispielsweise bei Nadelstichverletzung durch kontaminierte Kanülen, empfiehlt das Erklärvideo, den Blutfluss zu fördern, die Wunde zu desinfizieren und den Betriebsarzt zu kontaktieren.

Das Resümee: „Unter Beachtung der Standardhygienemaßnahmen wie dem Tragen von Arbeitskleidung, Schutzbrille, Mund- und Nasenschutz und Handschuhen und der üblichen Maßnahmen zur Reinigung und Desinfizierung nach der Behandlung besteht im Praxisalltag keine Ansteckungsgefahr."

Allerdings seien Diskretion und Datenschutz in solchen Fällen besonders wichtig für das Arzt-Patienten-Verhältnis.

Video unter www.bzaek.de/fuer-medien/video-audio.html

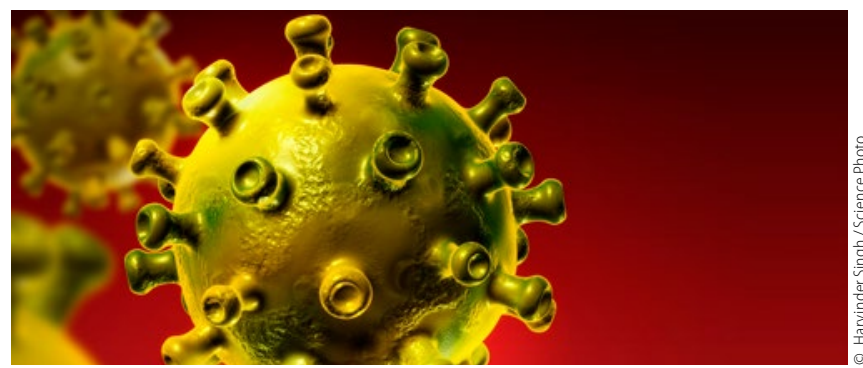

\title{
Jetzt bewerben
}

\section{Förderpreis für junge Wissenschaftler 2018}

Wer gerade an einem Forschungsprojekt arbeitet oder bereits eine wissenschaftliche Arbeit in der Zahnmedizin geschrieben hat, sollte sich jetzt für den Förderpreis 2018 beim Freien Verband Deutscher Zahnärzte (FVDZ) bewerben. Einsendeschluss ist der 31. Oktober 2017. Mit bis zu 10.000 Euro jährlich werden vielversprechende Arbeiten junger Wissenschaftler prämiert. Die Stiftung Zahnärztliche Wissenschaften verleiht in Zusammenarbeit mit dem FVDZ bereits seit 2002 jedes Jahr den Förderpreis. Die Preisverleihung findet traditionell bei den jährli- chen Sommer- oder Winterkongressen des FVDZ bei der jeweiligen Eröffnungsfeier statt. Die Gewinnerin des Förderpreises 2017, Dr. Ghazal Aarabi (Universitätsklinikum HamburgEppendorf), nahm den Förderpreis auf dem 49. Zahnärzte Winterkongress in Ischgl entgegen und berichtete dem interessierten Publikum von ihrer Arbeit zum Thema „Entzündungen der Mundhöhle als unabhängige Risikofaktoren für kardiovaskulare Erkrankungen“. Weitere Infos unter dem Stichwort „Förderpreis“ auf www.fvdz.de. red 